\title{
Paraneoplastic palmoplantar keratoderma revealing small cell lung cancer
}

\section{Sanae Sialiti, Sara Mai, Mariame Meziane, Nadia Ismaili, Laila Benzekri, Karima Senouci}

Department of Dermatology, University Hospital Ibn Sina Rabat, Morocco

Corresponding author: Dr. Sanae Sialiti, E-mail: sialiti2390@gmail.com

Sir,

A 62-year-old man with a history of 45 pack-years of smoking, presented to the dermatology department with progressive thickening of the palms and soles that had started for 2 years ago, with maximal involvement of the soles associated to onychogriphosis (Fig. 1). The patient exhibited no other lesions, notably no fine scale on the antihelix neither on the nose, and had no general symptoms. Initial symptomatic treatment was unsuccessful.

Histological examination of a skin biopsy revealed keratoderma features with no etiologic orientation on and laboratory tests showed elevated serum carcinoembryonic antigen level. A suspicious opacity was detected on chest X-ray. A CT-scan was then performed revealing a lung tumor (Fig. 2). The tumornode-metastasis (TNM) staging was T4N1M0, and CT-Guided biopsy concluded to a small cell lung carcinoma.

A diagnosis of paraneoplastic palmoplantar keratoderma associated with lung cancer was made and patient underwent mechanical keratolysis associated to chemotherapy with good results up today (Fig. 3).

Palmoplantar keratoderma (PPK) may be acquired or inherited. It is a dermatosis that presents with hyperkeratosis of the palms and soles. Ninety percent of acquired diffuse PPK is in association with visceral malignancies, such as esophageal, gastric, pulmonary, and bladder carcinomas [1]. It occurs more frequently in male patients, and in women, it has been linked to ovarian and breast carcinoma [2]. The pathogenesis of

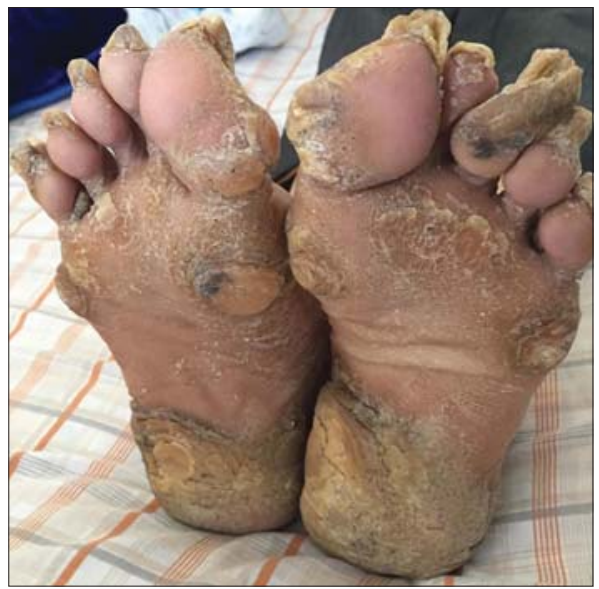

Figure 1: Acquired plantar keratoderma.

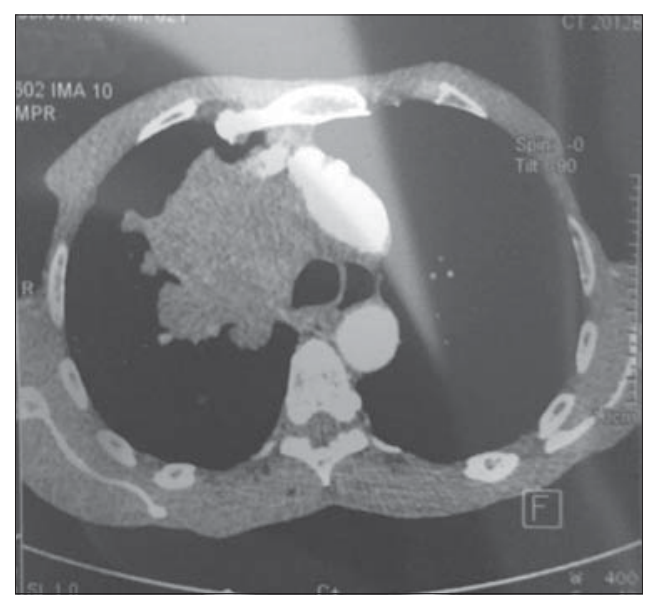

Figure 2: CT-scan revealing lung cancer.

palmoplantar keratoderma associated with pulmonary cancer is poorly understood.

Paraneoplastic palmoplantar keratoderma has to be differentiated from non-cancerous conditions, and the

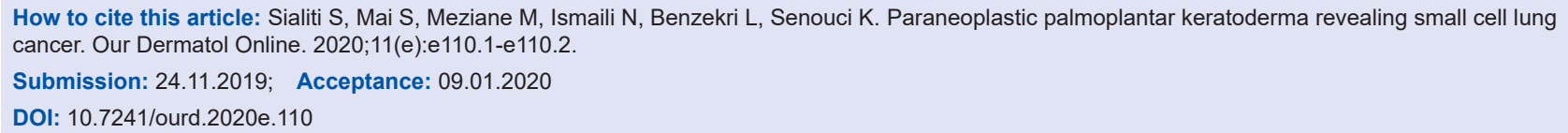


www.odermatol.com

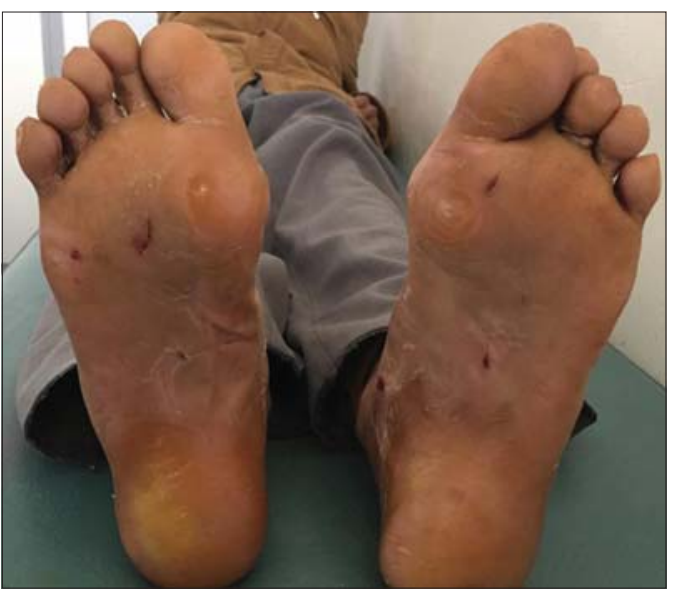

Figure 3: Resolution of skin findings after topical keratolytics and chemotherapy.

first step management of acquired PPK is to determine the etiology via a total-body skin examination, imaging and blood tests. However, it should not delay diagnosis of visceral malignancy and once the underlying neoplasia is treated, we notice a complete resolution of cutaneous findings.

\section{Consent}

The examination of the patient was conducted according to the Declaration of Helsinki principles.

The authors certify that they have obtained all appropriate patient consent forms. In the form the patient(s) has/have given his/her/ their consent for his/her/their images and other clinical information to be reported in the journal. The patients understand that their names and initials will not be published and due efforts will be made to conceal their identity, but anonymity cannot be guaranteed.

\section{REFERENCES}

1. KalliniJR, Sadeghani K, Khachemoune A. Paraneoplastic palmoplantar keratoderma secondary to metastatic uterine adenocarcinoma. Cutis. 2017;99:E32-5.

2. Vignale RA, Espasandín J, Paciel J, Scarrone V. [Diagnostic value of keratosis palmaris as indicative sign of visceral cancer]. Med Cutan Ibero Lat Am. 1983;11:287-92.

Copyright by Sanae Sialiti, et al. This is an open-access article distributed under the terms of the Creative Commons Attribution License, which permits unrestricted use, distribution, and reproduction in any medium, provided the original author and source are credited.

Source of Support: Nil, Conflict of Interest: None declared. 